\title{
Identification of a Novel NtLRR-RLK and Biological Pathways That Contribute to Tolerance of TMV in Nicotiana tabacum
}

\author{
Jing Wang, ${ }^{1}$ Fengsheng Hao, ${ }^{2}$ Kunfeng Song, ${ }^{2}$ Weihuan $\mathrm{Jin},{ }^{2} \mathrm{Bo} \mathrm{Fu},{ }^{1}$ Yuanfang Wei, ${ }^{2}$ Yongchun Shi, ${ }^{2}$ \\ Hongxiang Guo, ${ }^{2,+}$ and Weiqun Liu',2,+ \\ ${ }^{1}$ College of Tobacco Science, Henan Agricultural University, Zhengzhou, China \\ ${ }^{2}$ College of Life Sciences, Henan Agricultural University, Zhengzhou, China
}

Accepted 20 March 2020.

Tobacco mosaic virus (TMV) infection can causes serious damage to tobacco crops. To explore the approach of preventing TMV infection of plants, two tobacco cultivars with different resistances to TMV were used to analyze transcription profiling before and after TMV infection. The involvement of biological pathways differed between the tolerant variety (Yuyan8) and the susceptible variety (NC89). In particular, the plant-virus interaction pathway was rapidly activated in Yuyan8, and specific resistance genes were enriched. Liquid chromatography tandem mass spectrometry analysis detected large quantities of antiviral substances in the tolerant Yuyan8. A novel Nicotiana tabacum leucine-rich repeat receptor kinase (NtLRR-RLK) gene was identified as being methylated and this was verified using bisulfite sequencing. Transient expression of TMV-green fluorescent protein in pRNAi-NtLRR-RLK transgenic plants confirmed that NtLRR-RLK was important for susceptibility to TMV. The specific protein interaction map generated from our study revealed that levels of BIP1, E3 ubiquitin ligase, and LRR-RLK were significantly elevated, and all were represented at node positions in the protein interaction map. The same expression tendency of these proteins was also found in pRNAi-NtLRR-RLK transgenic plants at $24 \mathrm{~h}$ after TMV inoculation. These data suggested that specific genes in the infection process can activate the immune signal cascade through different resistance genes, and the integration of signal pathways could produce resistance to the virus. These results contribute to the overall understanding of the molecular basis of plant resistance to TMV and in the long term could identify new strategies for prevention and control virus infection.

\section{J. Wang and F. Hao contributed equally to this work.}

Data availability: The RNA-Seq data used in this study are freely available at the China National GeneBank short sequence archive under project accession CNP0000253.

${ }^{\dagger}$ Corresponding authors: W. Liu; wqliu@henau.edu.cn and H. Guo; guohongxiang06@126.com

*The $\boldsymbol{e}$-Xtra logo stands for "electronic extra" and indicates that one supplementary figure and six supplementary tables are published online.

The author(s) declare no conflict of interest.

๑) 2020 The American Phytopathological Society
Keywords: biological pathway, epigenetic, genomics, mechanisms of pathogenicity, metabolomics, pathogenesis-related proteins, plant defense mechanisms, plant resistance, protein interaction, proteomics, TMV, tolerance, transcriptome, virus-induced gene silencing

Tobacco mosaic virus (TMV) is a kind of intracellular parasitic-pathogenic microorganism which uses the host replicase system to complete its own replication. Currently, nongenetically modified organism methods are nearly unavailable for effectively preventing TMV infection; therefore, it remains a challenge to control tobacco mosaic disease (Han et al. 2014; Wang et al. 2013). To explore the approach of controlling TMV infection in plants, scientists have investigated the infection process from different fields, and exciting progress in research on host factors has been achieved (Wang 2015). Some host factors are involved in all major processes of viral infection (Wang and Krishnaswamy 2012). These results demonstrate that viral infection is the consequence of complex molecular interactions between host plants and viruses.

Except for the establishment of immune mechanisms triggered by pathogenic bacteria, including pathogen-associated molecular pattern (PAMP)-triggered immunity (PTI) and effector-triggered immunity (ETI), plant disease resistance is based on certain substances that can reset the metabolic pathways of infected plants. TMV, as a specialized intracellular parasite, completely depends on the host cell environment. This dependency requires the ability of viral proteins and nucleic acids to transform the normal function of host factors, which can affect the normal physiological and biochemical processes in plants. In this process, signal transduction induced by pathogen infection activates the expression of regulators or transcription factors, thereby resetting host biological processes and producing disease-resistant substances. Therefore, it is important to study the changes of host gene expression patterns and other symptom-related molecular events in the process of virus infection. The findings of each new host factor and the resetting of biological pathways will provide clues to the molecular basis of host susceptibility or disease symptoms.

In our previous work, it was demonstrated that tobacco varieties Yuyan8 and NC89 have different tolerances to TMV infection. Yuyan8 was obtained through selective breeding from NC89 during a tobacco mosaic disease outbreak in the field, and it displays tolerance to TMV infection. The resistance genes were markedly upregulated in Yuyan8, and the maintenance of the D1 protein and reasonable utilization of energy 
contributed to the resistance of the Yuyan8 variety (Wang et al 2016). However, the complex molecular biological relationships between virus and host interactions require systematic analysis. To reveal these problems, whole-transcriptome sequencing (RNA-seq) was carried out and combined with metabolomics and epigenetic analysis to explore complex molecular biological relationships between virus and host interactions. This helps us accumulate important data to lay the foundation for developing strategies to control or prevent viral infections.

\section{RESULTS}

Transcriptome assembly of Yuyan8, Yd, NC89, and Nd.

To explore the transcriptome changes caused by TMV infection in tobacco (Nicotiana tabacum), we assessed the global transcriptome profile of Yuyan8 and NC89 before and after TMV inoculation. RNA was extracted for RNA-seq analysis at $0 \mathrm{~h}$ (Yuyan8 and NC89) and $24 \mathrm{~h}$ postinfection (hpi) (Yd and Nd). In total, 56,805,878 (94.6\%), 62,147,726 (96.4\%), $80,023,184$ (96.8\%), and 94,555,612 (95.6\%) clean reads were obtained in the NC89, Yuyan8, Nd, and Yd libraries, respectively (Supplementary Table S1). The data indicated that the sequencing depth was sufficient for transcriptome coverage in tobacco. In total, $16 \mathrm{G}$ sequencing data were generated and de novo assembled into 72,105 unigenes with an average length of 828 bp (Supplementary Table S2). Sequence quality analysis revealed that these data were completely sufficient for the quantitative analysis of gene expression.

To confirm the expression of differentially expressed genes (DEGs), 12 differentially expressed unigenes were randomly selected for quantitative real-time (qRT)-PCR analysis from the unigene libraries. As expected, these genes had the same expression pattern as the DEG analysis, except for aquaporin (Fig. 1).

\section{Global transcriptome changes between Yuyan8 and NC89 after TMV inoculation.}

To investigate the difference between Yuyan8 and NC89 in response to TMV infection, the DEGs among the four libraries were revealed through pairwise comparison. There were 10,920 DEGs in Yuyan8 versus NC89 (5,838 upregulated and 5,082 downregulated), 7,572 DEGs in Yd versus Yuyan8 $(5,754$ upregulated and 1,818 downregulated), and 9,151 DEGs in $\mathrm{Nd}$ versus NC89 (6,006 upregulated and 3,145 downregulated) (Fig. 2A). The changes in gene expression in Yuyan8 were fewer than those in NC89 after TMV infection, suggesting that natural variation endowed Yuyan8 with the characteristic of insensitivity to TMV infection.

Due to the same genetic background between Yuyan8 and NC89, the commonality and specificity analysis of DEGs between Yd versus Yuyan8 and Nd versus NC89 was helpful to reveal the molecular basis of tolerance to TMV infection. There were 4,739 unique genes in Yd versus Yuyan8 and 6,318 unique genes in Nd versus NC89 (Fig. 2B). Differentiation of unique genes in the Yuyan8 and NC89 varieties implied their differences in responding to TMV infection and resetting biological pathways.

To obtain a functional overview of differential genes in the Yuyan8 and NC89 varieties, 4,739 and 6,318 DEGs, respectively, were analyzed by Kyoto Encyclopedia of Genes and Genomes (KEGG) pathway enrichment (Fig. 2C and E). The terms "plant-pathogen interaction", "glutathione metabolism", "isoflavonoid biosynthesis", and "glutathione metabolism" were high-reliability terms in Yd versus Yuyan8 (Fig. 2C). Meanwhile, the terms "isoflavonoid biosynthesis", "photosynthesis-antenna proteins", "pyrimidine metabolism", and "tyrosine metabolism" were enriched in Nd versus NC89 (Fig. 2E). The term "isoflavonoid biosynthesis" pathway was enriched in both $\mathrm{Nd}$ versus NC89 and Yd versus Yuyan8 but the genes of this term were different between Nd versus NC89 and Yd versus Yuyan8, and half of the genes were downregulated in Nd versus NC89. This allowed us to explore the global activation of specifically metabolic pathways responding to TMV infection in the two varieties.

\section{Unique DEGs of Yd in response to TMV infection.}

Plant-pathogen interaction involves the activation of signals, which results in a rapid defense response. This response helps
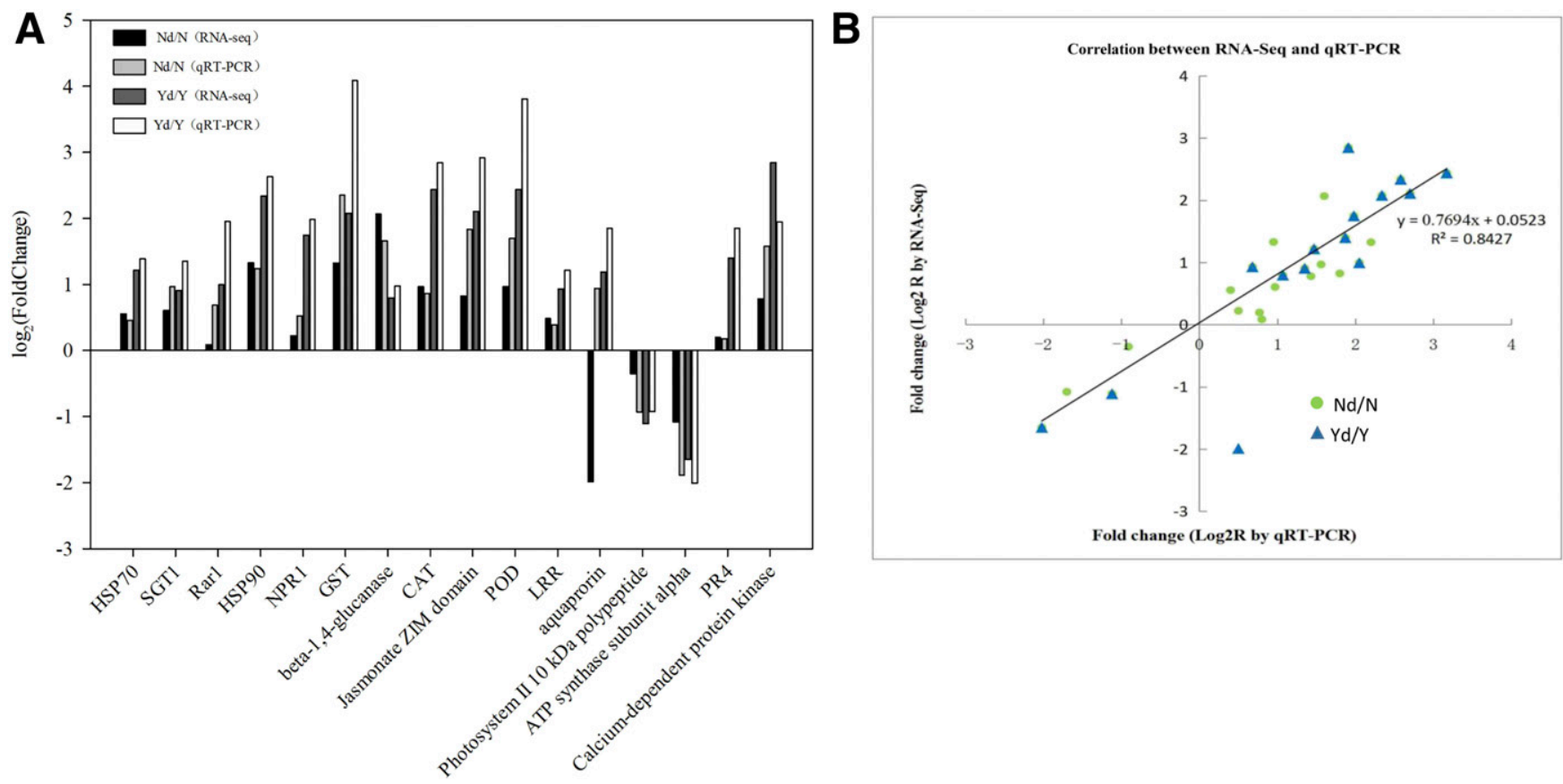

Fig. 1. Validation of the whole-transcriptome sequencing (RNA-seq) results by quantitative real-time $(q R T)-P C R$. Varieties $N=$ NC89, $Y=$ Yuyan8, and Nd and $\mathrm{Yd}=24 \mathrm{~h}$ postinfection. A, Expression levels of 12 randomly selected genes from RNA-seq and qRT-PCR results. B, Correlation analysis of RNA-seq and qRTPCR results. 
A

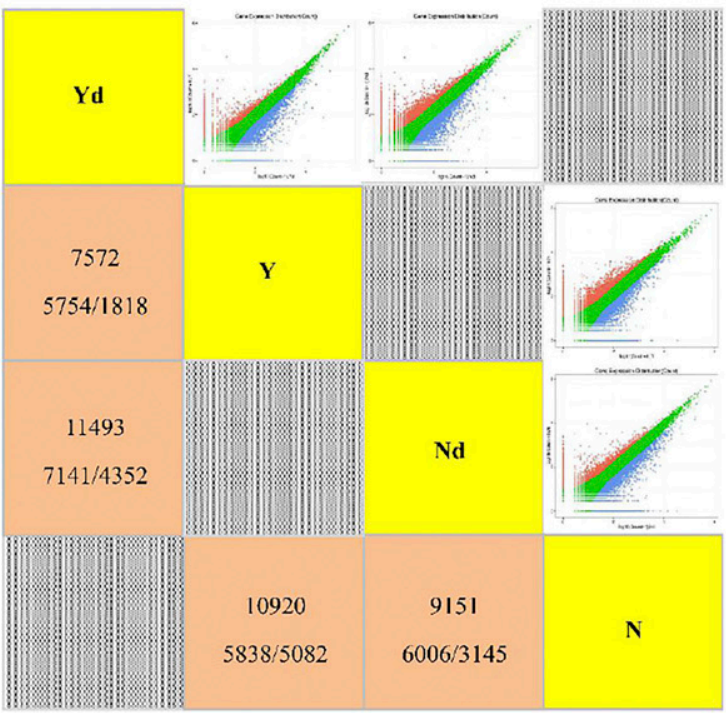

C

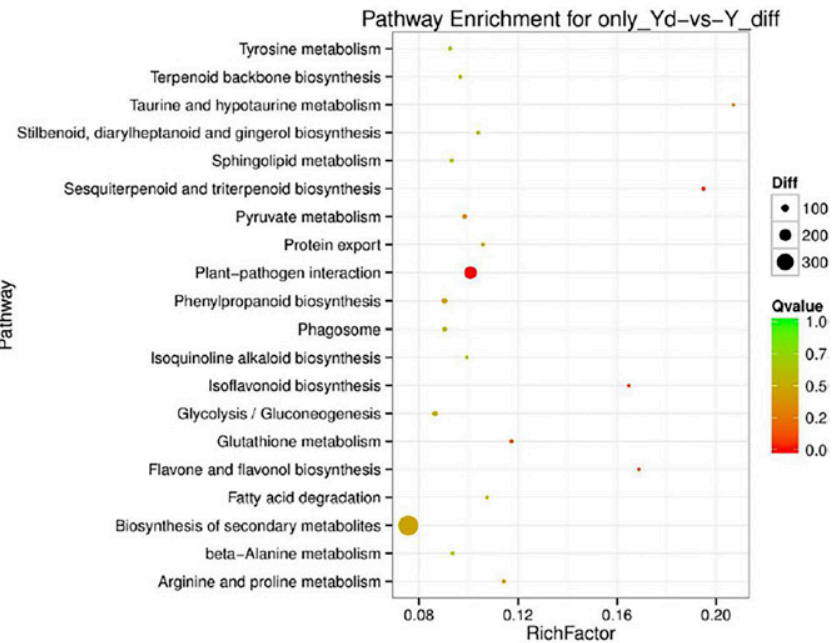

E

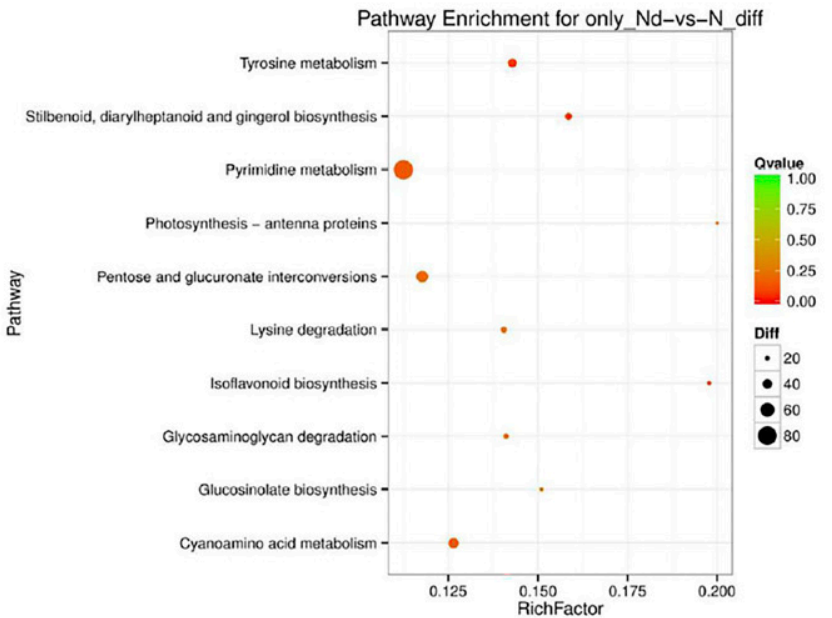

\section{B Yd vs Y Nd vs N}

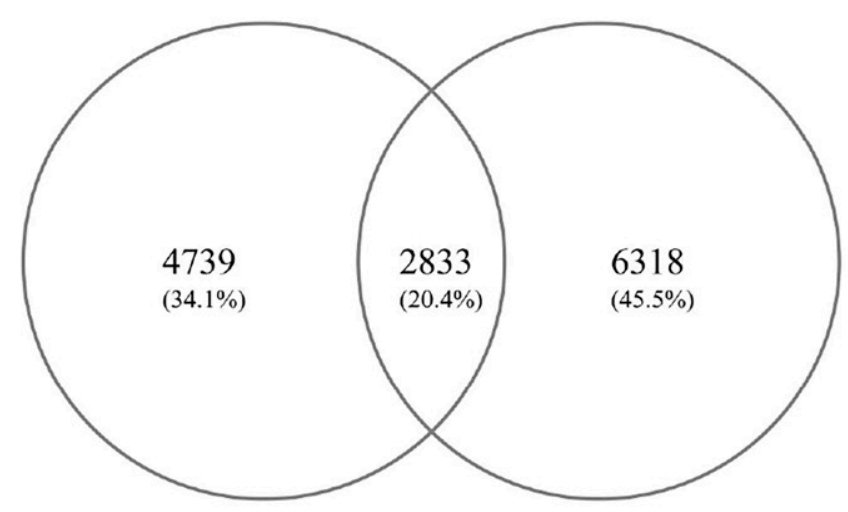

D

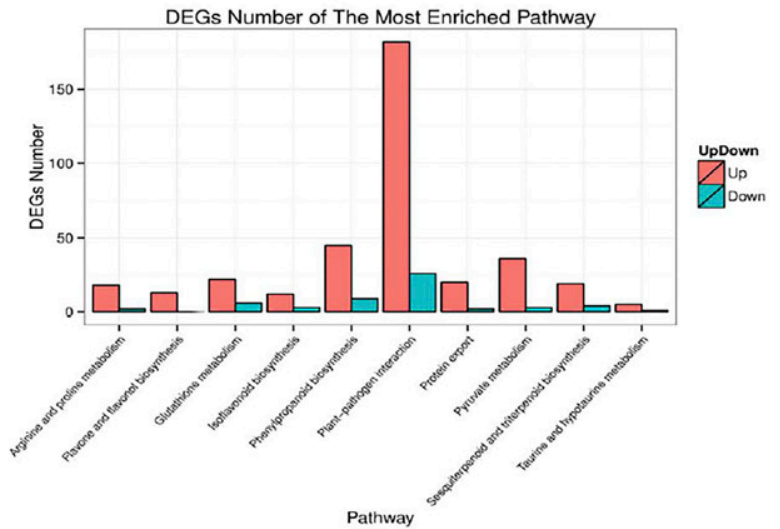

$\mathbf{F}$

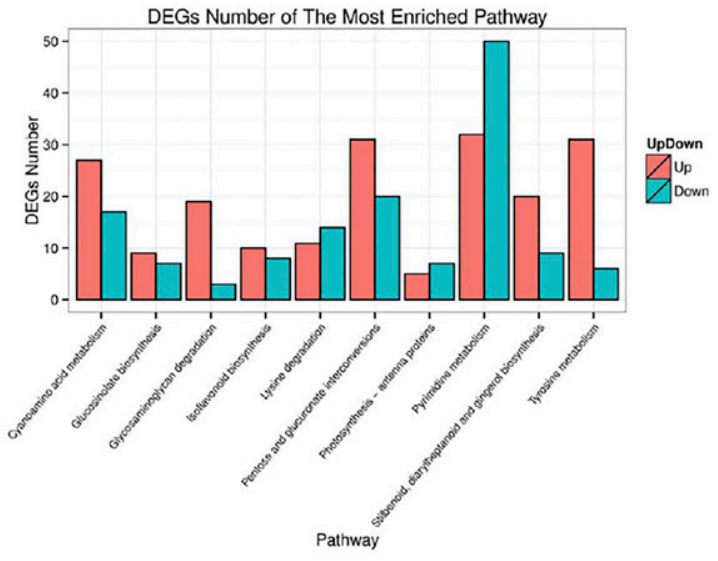

Fig. 2. Screening and analysis of differentially expressed genes in the four samples. A, Scatter plots and comparisons of differentially expressed genes (DEGs) for Yd, Yuyan8 (Y), Nd, and NC89 (N). Values represent the number of differential unigenes (up- and downregulated) between materials pairings. B, The Venn diagram demonstrates the common and unique DEGs between Yd versus Y and Nd versus N. C and $\mathbf{E}$, The enrichment pathway of DEGs in 4,973 and 6,318, respectively. $\mathbf{D}$ and $\mathbf{F}$, The number of genes in the enrichment pathway (red indicates upregulation and blue indicates downregulation). The X-axis label represents RichFactor (RichFactor $=$ the ratio between counts of differentially expressed genes and all annotated genes enriched in a pathway), and the Y-axis label represents the pathway $(\mathrm{Q}<1.0)$. 
the plant host to avoid further infection of the pathogens. Notably, the 208 unigenes in the plant-pathogen interaction pathway included 38 WRKY transcription factors, 13 leucinerich repeat (LRR) receptor-like serine/threonine-protein kinase, 10 resistance $(\mathrm{R})$ proteins, and 30 calcium-binding or calmodulin proteins (Supplementary Table S3). The function of these genes should be the basis of tolerance to TMV infection in Yuyan8.

WRKY, an important transcription factor gene family, is involved in the response to environmental signals and the regulation of plant growth and development (Agarwal et al. 2011; Bhattarai et al. 2010; van Verk et al. 2011). It has been shown that PTI and ETI can induce the expression of most WRKY transcription factors. After tobacco infection with TMV, the expression of WRKY genes was increased in leaves (Ren et al. 2010). In transgenic plants overexpressing WRKY genes, the expression of pathogenesis-related (PR) proteins could be increased to enhance resistance to phytopathogens (Choi et al. 2015; Li et al. 2015; van Verk et al. 2008).

The host signal defense system can be induced by $\mathrm{R}$ proteins, which usually contain LRRs in plants. The LRR proteins have diverse functions in developmental processes, signal transduction, and defense-related pathways (Feng et al. 2012; Torii 2004). LRRs are mainly involved in recognizing the plant innate immune system (Takken et al. 2006). There were 18 LRRkinase receptor genes that responded to TMV infection in Yuyan8, 13 of which belonged to flagellin receptor FLS2 (Robatzek et al. 2006). FLS2, a putative receptor kinase, is ubiquitously expressed. The FLS2 gene shared structural and functional homologies with known plant resistance genes in the innate immune system (Dodds and Rathjen 2010). Interestingly, 28 DEGs were enriched in the glutathione metabolism pathway in Yd versus Yuyan8 $(4,739)$ but this was not found in Nd versus NC89 (6,318). The glutathione metabolism pathway was also enriched in 2833 DEGs that overlapped between Yd versus Yuyan8 and Nd versus NC89 (Supplementary Fig. S1). Yuyan8 had stronger responses in the glutathione metabolism pathway than NC89 after TMV infection, which had been confirmed by Huang et al. (2017), indicating that the higher levels of glutathione-S-transferases (GSTs) protected against dangerous molecules generated by TMV attack in Yuyan8. Indeed, except for GST, the enriched glutathione metabolism pathway also included some peroxidases such as glutathione peroxidase and L-ascorbate peroxidase which all contribute to the elimination of reactive oxygen in plants. Glucose-6-phosphate 1-dehydrogenase can provide NADPH for ascorbate peroxidase to detoxify excess $\mathrm{H}_{2} \mathrm{O}_{2}$ (Tanaka et al. 2014). Accumulation of reactive oxygen species scavenging enzymes was also found in cross-protected plants, providing rescues from excessive oxidative damage (Das et al. 2019). These results implied that the GST activity could be improved to increase the content of glutathione in Yuyan8, which contributed to the tolerance of Yuyan8 to TMV infection.

\section{Verification of the differences in flavonoid compounds between the Yuyan8 and NC89 varieties through metabolomics analysis.}

Flavonoid biosynthesis was one of the enriched pathways of the specifically expressed genes in both varieties after TMV infection. However, the majority of genes in Yuyan8 were upregulated, and the opposite was observed in NC89. Many flavonoids have potential value as antiviral drugs. To further verify the difference and determine novel constituents that can be used as a target for improving the resistance of tobacco plants, the same set of materials used for transcriptome sequencing was used for the targeted metabolomics analysis. In total, 1,204 to 1,458 peaks were detected in each sample. To find the metabolites influenced by TMV infection, the significant analysis of variance of test groups was carried out with the Duncan test; the results are shown in Table 1. The accumulation of most flavonoid metabolites was significantly increased in Yd, such as "6-hydroxy-rutin", "5,7-di-O-methylquercetin", and "astragalin" (Fig. 3). These flavonoid metabolites have antiviral activity and can also effectively inhibit the production of free radicals (Singh et al. 2017), lower the concentration of peroxides, and protect tissue from oxidative damage (Bergman et al. 2003).

\section{Dynamic changes in methylation in Yuyan8 and NC89 after TMV inoculation.}

Comprehensive analysis of the transcriptome and metabolome showed that there were substantial changes in the DEGs between Yuyan8 and NC89 after TMV inoculation, and these changes were the consequence of plant adaptation to biotic stress by the alteration of biological pathways. Although the trigger of the defense signaling is unknown, increasing evidence suggests that the plant genome has the ability to respond sensitively and dynamically to diverse stresses by changing DNA methylation patterns (Akimoto et al. 2007; Boyko et al. 2007; Finnegan et al. 1993; Sha et al. 2005). Yuyan8 was obtained through pedigree selection from the NC89 population during a TMV outbreak; therefore, it was possibly produced by natural variation under such environmental pressure. However, whether this event was related to DNA methylation was unknown; thus, the methylation level of the genomes in the two varieties was examined at 12 h, 24 h, 6 days, and 9 days after TMV infection with the methyl-sensitive amplified polymorphism (MSAP) technique.

Table 1. Differential metabolites identified by tandem mass spectrometry (MS/MS) from the tobacco leaves ${ }^{\mathrm{y}}$

\begin{tabular}{|c|c|c|c|c|c|c|c|c|c|c|}
\hline \multirow[b]{2}{*}{ Index } & \multirow[b]{2}{*}{ Compounds } & \multicolumn{2}{|c|}{ Molecular } & \multirow{2}{*}{$\begin{array}{l}\text { Time } \\
(\mathbf{m i n})\end{array}$} & \multirow[b]{2}{*}{ Mass (ppm) } & \multirow[b]{2}{*}{ MS/MS fragments } & \multicolumn{4}{|c|}{ Relative amount ${ }^{\mathrm{z}}$} \\
\hline & & Formula & Weight & & & & $\mathbf{N}$ & Nd & $\mathbf{Y}$ & Yd \\
\hline X300 & Iso-Kaemferide & $\mathrm{C} 16 \mathrm{H} 12 \mathrm{O} 6$ & 300.06 & 9.11 & $299.0554(-2.5)$ & $2,99,28,52,55,227$ & $1.01 \mathrm{~b}$ & $1.12 \mathrm{~b}$ & $1.2 \mathrm{~b}$ & $2.1 \mathrm{a}$ \\
\hline X316 & Quercetin 3-methyl ether & $\mathrm{C} 16 \mathrm{H} 12 \mathrm{O} 7$ & 316.06 & 8.71 & $315.0506(-1.3)$ & $3,15,30,02,71,25,52,43,000$ & $6.15 \mathrm{~b}$ & $6.12 \mathrm{~b}$ & $10.16 \mathrm{ab}$ & $13.58 \mathrm{a}$ \\
\hline X330 & 5,7-Di-O-methylquercetin & $\mathrm{C} 17 \mathrm{H} 14 \mathrm{O} 7$ & 330.07 & 9.17 & $329.0659(-2.5)$ & $32,93,14,29,92,85,27,10,00,000$ & $7.78 \mathrm{~b}$ & $10.09 \mathrm{~b}$ & $13.39 \mathrm{~b}$ & $19.7 \mathrm{a}$ \\
\hline X448 & Astragalin & $\mathrm{C} 21 \mathrm{H} 20 \mathrm{O} 11$ & 448.10 & 7.24 & $447.0923(-2.3)$ & $4,47,28,52,55,227$ & $2.72 \mathrm{~b}$ & $3.42 \mathrm{ab}$ & $4.24 \mathrm{ab}$ & $4.85 \mathrm{a}$ \\
\hline X464 & Isoquercetin & $\mathrm{C} 21 \mathrm{H} 20 \mathrm{O} 12$ & 464.10 & 6.86 & $463.0876(-1.3)$ & $4,63,30,02,71,25,52,43,000$ & $22.72 b$ & $26.98 \mathrm{ab}$ & $30.33 \mathrm{ab}$ & $41.28 \mathrm{a}$ \\
\hline X626 & 6-Hydroxy-rutin & $\mathrm{C} 27 \mathrm{H} 30 \mathrm{O} 17$ & 626.15 & 6.69 & $625.1392(-3)$ & $62,53,16,29,92,71,255$ & $3.45 \mathrm{~b}$ & $4.09 \mathrm{~b}$ & $6.57 \mathrm{ab}$ & $9.73 \mathrm{a}$ \\
\hline X772 & $\begin{array}{l}\text { Quercetin-3,7- } \\
\text { rutinosogalactoside }\end{array}$ & $\mathrm{C} 33 \mathrm{H} 40 \mathrm{O} 21$ & 772.21 & 5.03 & $771.1982(-0.9)$ & $7,71,60,94,62,30,02,71,000$ & $3.85 \mathrm{~b}$ & $4.74 \mathrm{ab}$ & $5.21 \mathrm{a}$ & $6.07 \mathrm{a}$ \\
\hline X165 & Phenylalanine & $\mathrm{C} 9 \mathrm{H} 11 \mathrm{NO} 2$ & 165.08 & 2.19 & $164.0717(-0.9)$ & $164,147,103,72$ & $4.35 \mathrm{c}$ & $5.18 \mathrm{bc}$ & $8.68 \mathrm{ab}$ & $10.58 \mathrm{a}$ \\
\hline X338 & Coumaryl quinic acid & $\mathrm{C} 16 \mathrm{H} 18 \mathrm{O} 8$ & 338.10 & 6.46 & $337.0923(-2.5)$ & $337,191,173,163,119,93$ & $32.79 \mathrm{~b}$ & $40.43 \mathrm{~b}$ & $59.73 \mathrm{ab}$ & $101.48 \mathrm{a}$ \\
\hline
\end{tabular}

y Time = retention time and Mass = experimental mass.

z Varieties $\mathrm{N}=\mathrm{NC} 89, \mathrm{Y}=$ Yuyan8, and $\mathrm{Nd}$ and $\mathrm{Yd}=24 \mathrm{~h}$ postinfection. Lowercase letters denote the significant differences from Duncan's multiple range tests with $P<0.05$ 
MSAP was performed with 34 combinations of EcoRI (E) and HpaII/MspI (H/M) primer pairs (Supplementary Table S4). The results showed that methylated loci gradually increased from $0 \mathrm{~h}$ to 6 days after TMV infection and then decreased in NC89. Compared with NC89, the methylated loci observably increased from 0 to $24 \mathrm{~h}$ and subsequently decreased in Yuyan 8 (Table 2). Except for the nonmethylated loci, the MSAP markers were observed in $163(27.03 \%$ of all loci), 167 (27.65\% of all loci), 176 (29.29\% of all loci), 179 (29.73\% of all loci), and 177 (29.31\% of all loci) methylated loci in NC89 at 0 h, 12 h, 24 h, 6 days, and 9 days, respectively, after TMV inoculation. In Yuyan8, the MSAP markers were found in 162 (26.91\% of all loci), 176 (29.33\% of all loci), 179 (29.68\% of all loci), 176 (29.18\% of all loci), and 173 (29.79\% of all loci) methylated loci at $0 \mathrm{~h}, 12 \mathrm{~h}, 24 \mathrm{~h}, 6$ days, and 9 days, respectively, after TMV inoculation. The increase in the methylation level at the early stage after TMV infection may be beneficial to the stability of the plant genome, and the decrease in the methylation level at the later stage after TMV infection may be conducive to the expression of resistance genes (Wada et al. 2004).

\section{Verification of polymorphic methylated sites by bisulfite sequencing PCR.}

To confirm the methylated loci obtained by MSAP, 30 polymorphic methylated fragments were sequenced, and six annotated loci were obtained by searching GenBank (Supplementary Table S5). The polymorphism sites of the LRR receptor kinase (RLK) were analyzed by bisulfite sequencing PCR (BSP). The methylation status of the LRR gene was higher in Nd than in NC89, whereas it was lower in Yd than in Yuyan8 (Fig. 4B). Interestingly, at cytosine guanine (CpG) loci 130 of the LRR, there was no methylation in Yuyan8 before and after TMV infection but the methylation level was very high in NC89 and Nd (Fig. 4B). Additionally, the expression level of LRR in NC89 and Nd was lower than that in Yuyan8 and Yd (Fig. 4C), indicating that the hypomethylation of the resistance-related $L R R$ gene is the reason for its high expression in the resistant
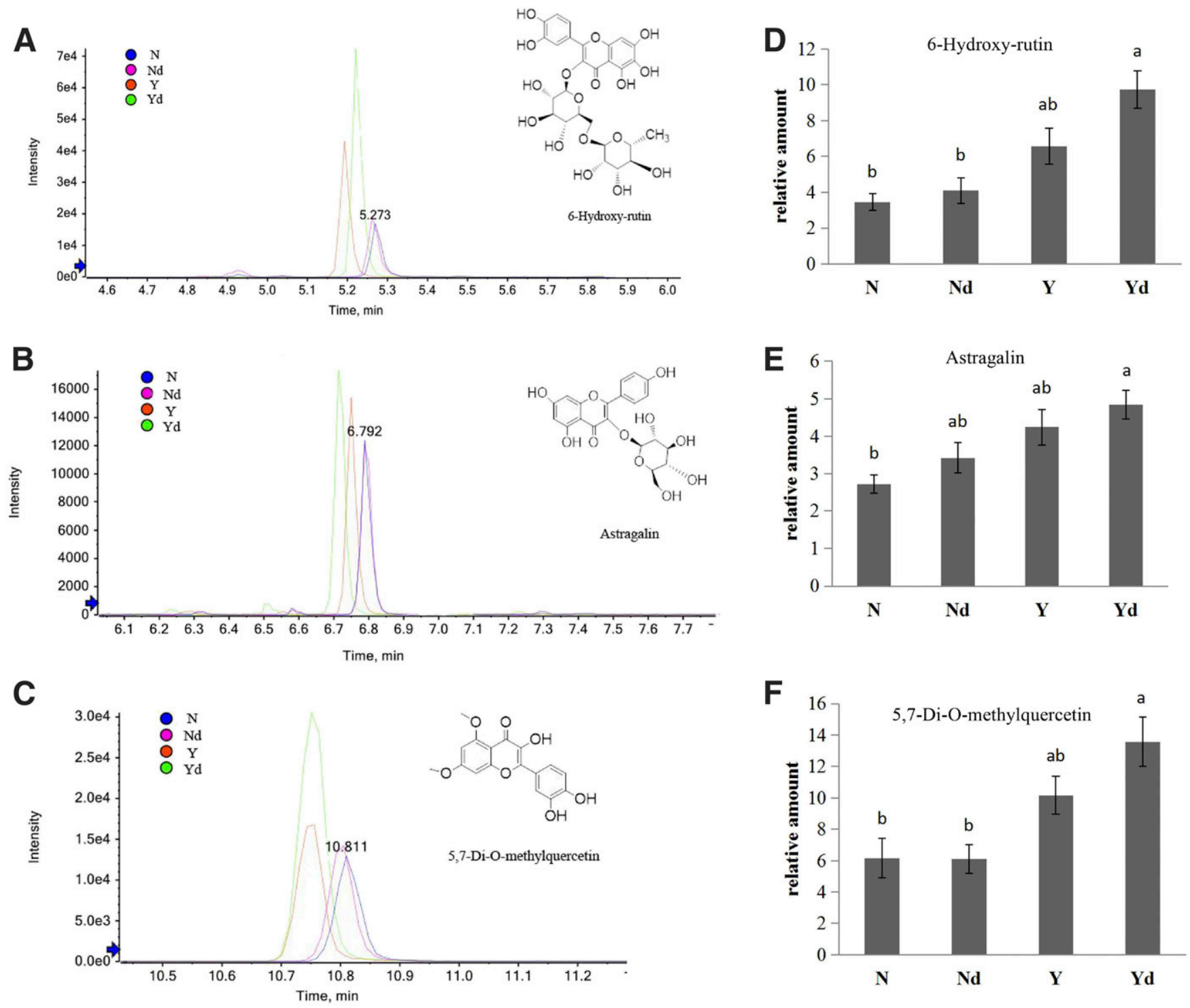

Fig. 3. Variation in flavonoid compounds in tobacco leaves in the four samples. Varieties $\mathrm{N}=\mathrm{NC} 89, \mathrm{Y}=\mathrm{Yuyan} 8$, and $\mathrm{Nd}$ and $\mathrm{Yd}=24 \mathrm{~h}$ postinfection. A to C, Ultraperformance liquid chromatography-quantitative time of flight chromatograms of 6-hydroxy-rutin, astragalin and 5,7-di-O-methylquercetin. D to F, Relative amount (peak areas) of 6-hydroxy-rutin, astragalin, and 5,7-di-O-methylquercetin in four samples. Repeated measures analysis of variance and Duncan's multiple comparison tests were used for statistical analysis. 
variety. The above results suggest that DNA methylation resets the biological pathway by affecting gene expression in plants to adapt to virus infection, while LRR demethylation is beneficial to activate or enhance gene expression for improving disease resistance.

To more clearly show the effect of methylated LRR-RLKs on NC89 species, their interaction with TMV-infection-responsive proteins was discussed. In Yuyan8, the LRR-RLKs were associated with endoplasmic reticulum (ER) stress. The expression level of ER luminal binding protein 1 (BIP1) was improved up to 700 times, which was related to the folding of secretory proteins in the ER. Meanwhile, the expression of the E3 ubiquitin-protein ligase KEG (E3-KEG) increased 70-fold, and it transmitted the signal of TMV infection directly to the intracellular calcium signal system jointly with other RLKs, improving the metabolism of isoflavones, flavonoids, phenylpropanoid, and glutathione through the activation of the transcription factor WRKY26 (Fig. 5A). In NC89, most TMVinfection-responsive genes were downregulated. Several key nodes showed that the DNA-directed RNA polymerase (DdRP) gene affected the expression of genes related to histone methylation, while serine hydroxymethyl transferase (SHM1/2) was involved in controlling cell damage caused by biotic and abiotic stress. Ultimately, glycosaminoglycan degradation was regulated (Fig. 5B). Interestingly, metabolism pathways are associated with the incidence of disease.

\section{Silencing of $N$. tabacum LRR receptor-like kinase significantly enhanced TMV infection.}

The above results showed that LRR $N$. tabacum $L R R-R L K$ $(N t L R R-R L K)$ surface receptor methylation in NC89 affected the susceptibility to TMV. To further confirm this result, the $p R N A i-N t L R R-R L K$ transgenic plants were created and transient expression of TMV-green fluorescent protein (GFP) by agroinfiltration showed strong viral fluorescence at 5 days compared with the wild type (Fig. 6A). Furthermore, the expressions of the critical node genes in the protein interaction network were detected in $p R N A i-N t L R R-R L K$ transgenic plants and the wild type at $24 \mathrm{~h}$ after inoculation with TMV (Fig. 6B). The results showed the same trend as the digitally expressed genes, suggesting that NtLRR-RLK methylation confers susceptibility to TMV by potentially activating the plant-pathogen interaction pathway in $N$. benthamiana.

\section{DISCUSSION}

The infection and reproduction of viruses are completely dependent on the host cell environment. Previous studies found

Table 2. Methylation pattern analyzed per $E c o \mathrm{RI}$ and $M s p \mathrm{I} / \mathrm{HpaII}$ primer combination

Number of loci $(\%)$

Cultivar, time Total Nonmethylated Hemimethylated Methylated

\begin{tabular}{lllll}
\hline $\begin{array}{l}\text { Yuyan8 } \\
0 \mathrm{~h}\end{array}$ & 602 & $440(73.09)$ & $62(10.30)$ & $100(16.61)$ \\
$12 \mathrm{~h}$ & 600 & $424(70.67)$ & $69(11.50)$ & $107(17.83)$ \\
$24 \mathrm{~h}$ & 603 & $424(70.32)$ & $72(11.94)$ & $107(17.74)$ \\
6 days & 603 & $427(70.81)$ & $71(11.77)$ & $105(17.41)$ \\
9 days & 601 & $428(71.21)$ & $70(11.65)$ & $103(17.14)$ \\
$\mathrm{NC} 9$ & & & & \\
$0 \mathrm{~h}$ & 603 & $440(72.97)$ & $63(10.45)$ & $100(16.58)$ \\
$12 \mathrm{~h}$ & 604 & $437(72.35)$ & $67(11.09)$ & $100(16.56)$ \\
$24 \mathrm{~h}$ & 601 & $425(70.72)$ & $70(11.65)$ & $106(17.64)$ \\
6 days & 602 & $423(70.27)$ & $72(11.96)$ & $107(17.77)$ \\
9 days & 604 & $427(70.70)$ & $68(11.26)$ & $109(18.05)$ \\
\hline
\end{tabular}

a link between the changes in host gene expression and sensitivity to TMV during infection. However, most of these studies analyzed a single pathway and host factor, and many causal relationships are unclear. Therefore, we must deeply understand the influence of virus infection on host factors, the reset of biological pathways, and the subsequent production of the resistant molecular cargoes. The two tobacco varieties Yuyan 8 and NC89 share the same genetic background but have different tolerances to TMV infection. Therefore, they are better experimental materials for elaborating the molecular mechanism of the host response to TMV infection. The integrative analysis of transcriptome, metabolome, and epigenetics suggested that the resistance of Yuyan8 to TMV infection originated from the signal recognition of viral invasion, which rapidly activated the internal immune system. In contrast, the high methylation of LRR in NC89 partially weakened the ability to transmit an extraneous signal inside the cell and activate the internal immune system.

\section{Yuyan8 can effectively recognize viral invasion and rapidly activate the internal immune system.}

In Yuyan8, TMV-infection-responsive genes included 38 WRKY transcription factors, 18 LRRs, 10 R proteins, and 34 calcium-binding or calmodulin proteins. The interaction of these proteins (Fig. 5A) showed that resistance to TMV resulted from the combination of multigene regulation. Notably, the
A
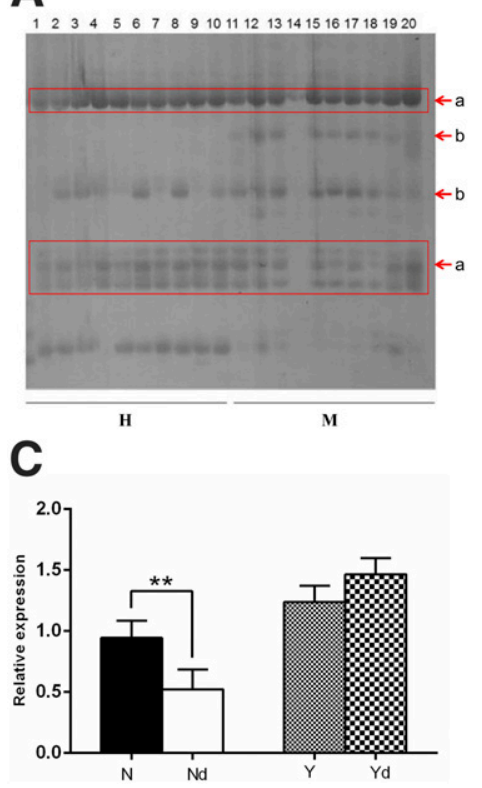

B

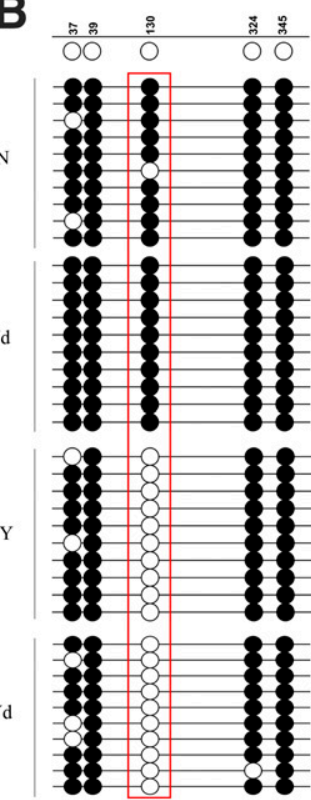

Fig. 4. Dynamic changes in and verification of the methylation loci. A, Methyl-sensitive amplified polymorphism (MSAP) bands generated by selective amplification with primer-pair combination $\mathrm{E}(\mathrm{ACC})+\mathrm{H} / \mathrm{M}$ (TTA). $\mathrm{H}$ or M represent genomic DNA samples digested by EcoRI/HpaII or $E c o \mathrm{RI} / M s p \mathrm{I}$, respectively. The presence of MSAP fragments in both $\mathrm{H}$ and $\mathrm{M}$ profiles indicated nonmethylated loci. The presence of $\mathrm{M}$ fragments and the absence of $\mathrm{H}$ fragments identified the methylated loci. The presence in $\mathrm{H}$ fragments but absence in $\mathrm{M}$ fragments was counted as hemimethylated loci. Row a indicates nonpolymorphic MSAP band patterns and row b indicates polymorphic MSAP markers. Lanes 1 to 5 and 11 to $15: 0 \mathrm{~h}, 12 \mathrm{~h}$, $24 \mathrm{~h}, 6$ days, and 9 days in NC89 after tobacco mosaic virus (TMV) infection; lanes 6 to 10 and 16 to $20: 0 \mathrm{~h}, 12 \mathrm{~h}, 24 \mathrm{~h}, 6$ days, and 9 days in Yuyan8 after TMV infection. B, Verification of methylation polymorphic loci in the four samples. Varieties $\mathrm{N}=\mathrm{NC} 89, \mathrm{Y}=$ Yuyan8, and $\mathrm{Nd}$ and $\mathrm{Yd}=$ $24 \mathrm{~h}$ postinfection. Each row represents a clone. The black circle represents methylation loci and the white circle represents nonmethylation loci. C, Gene expression level of leucine-rich repeat receptor-like serine/threonineprotein kinase. Data represent the means of three standard errors of the mean. Asterisks * and $* *$ indicate $P<0.05$ and 0.01 , respectively. 


\section{A}

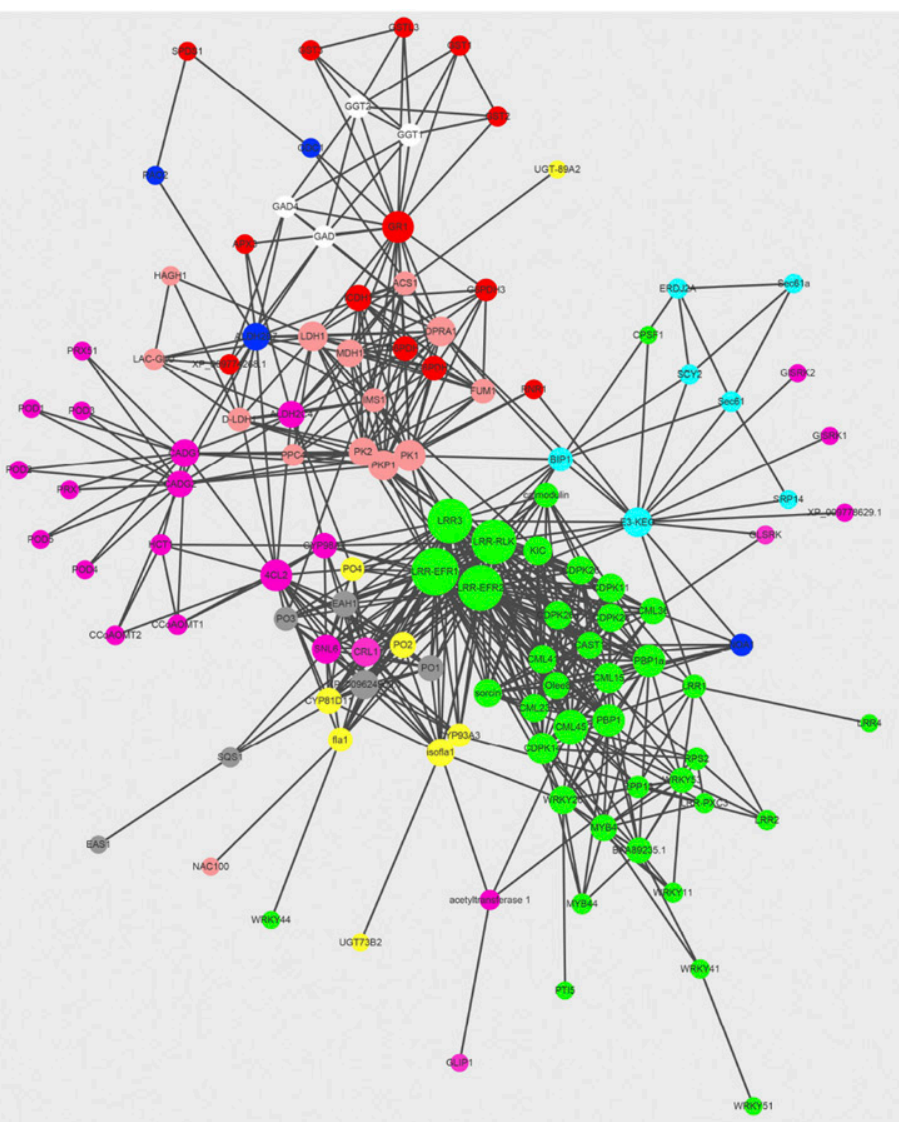

Glutathione metabolism

Isoflavonoid, Flavone and flavonol biosynthesis Plant-pathogen interaction

Protein export

Phenylpropanoid biosynthesis

Arginine and proline metabolism

Pyruvate metabolism

Sesquiterpenoid and triterpenoid biosynthesis

Taurine and hypotaurine metabolism
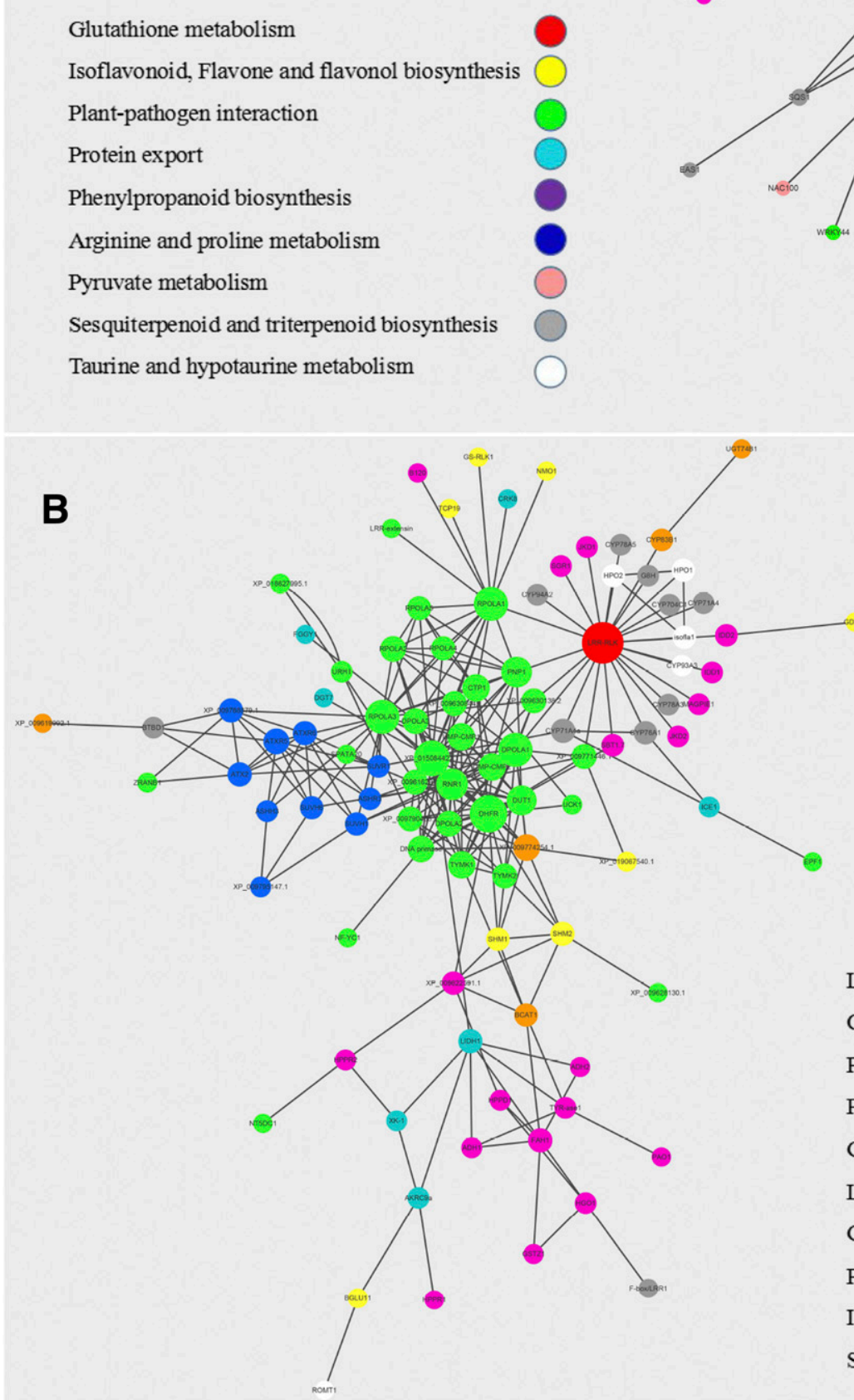

LRR-RLK

Cyanoamino acid metabolism

Pyrimidine metabolism

Pentose and glucuronate interconversions

Glucosinolate biosynthesis

Lysine degradation

Glycosaminoglycan degradation

Photosynthesis - antenna proteins

Isoflavonoid biosynthesis

Stilbenoid, diarylheptanoid and gingerol biosynthesis

Fig. 5. Cytoscape plugin analysis view of the protein interaction relationship in Yuyan8 and NC89. A, Sample Yuyan8. B, Sample NC89. The size of each node indicates the number of correlated genes. The color of nodes represents the metabolic pathway. 
expression of LRR in NC89 and Nd was lower than that in Yuyan8 and Yd, suggesting that Yuyan8 can effectively recognize viral invasion and rapidly activate the internal immune system.

The LRR-RLKs play an important role in plant immunity (Gao and Xue 2012). Many RLKs and receptor-like proteins act as pattern recognition receptors (PRRs) in plants. LRR-RLKs and LRR-receptor-like serine/threonine-protein kinase elongation factor Tu receptors all belong to PRRs, and they can perceive PAMPs and convert extracellular signals into intracellular signals (Gao and Xue 2012; Padmanabhan et al. 2009, 2013; Tang et al. 2017). As the second messenger, $\mathrm{Ca}^{2+}$ can indirectly trigger and lead to PTI (Sello et al. 2016; Zipfel 2014). R proteins can also trigger disease resistance. WRKY and MYB transcription factors play a vital role in these immune responses (Adachi et al. 2015; Bhattarai et al. 2010). WRKY transcription factors are potential regulatory factors in the pathogen resistance process and maintain the homeostasis of redox in cells by activating the glutathione system and flavonoid biosynthesis and increasing the synthesis of plant flavonoid antitoxins (Datta et al. 2015; Serrano et al. 2012). WRKY26 was located at a downstream node of the $\mathrm{Ca}^{2+}-\mathrm{CaM}$ messenger system and was regulated by MYB4 and MYB44. Here, the GST activity and glutathione content markedly increased in Yuyan8 after infection but it was not observed in NC89. In addition, the flavonoid antitoxin content in $\mathrm{Yd}$ was significantly higher than that in $\mathrm{Nd}$.

It is noteworthy that LRR-RLKs directly affect the activity of protein chaperones such as BIP1 and E3-KEG in the ER. ER stress occurs when homeostasis is disrupted by the accumulation of malformed proteins, and the unfolded protein response (UPR) is activated (Bravo et al. 2013; Verchot 2014, 2016). The UPR mechanism involves the increase in several ER-resident proteins such as calreticulin, protein disulfide isomerase, calmodulin, and BIP, which are required to restore proper protein folding (Ye et al. 2011). BIP belongs to the heat shock protein 70 (Hsp70) family that acts as a sensor of the ER stress response and provides suitable conditions for the production of secretory proteins by alleviating ER stress (Ohta et al. 2013). It has been reported that the expression of Hsp70 was increased in both $N$. benthamiana and Arabidopsis after virus infection (Aparicio et al. 2005). Interestingly enough, in addition to reducing the levels of unfolded protein, increasing evidence has shown that BIP facilitates host defenses by stabilizing immune receptors (Williams et al. 2014). E3-KEG assists ubiquitin in binding target proteins and promotes their degradation $(\mathrm{Gu}$ and Innes 2011). It has been reported that E3 Ub-ligases are involved in plant immune responses (Duplan and Rivas 2014). Therefore, in the protein interaction diagram of Yuyan8 (Fig. 5A), HIBI and E3-KEG were enriched at an evident node position, which further confirms the role of the BIP and E3-KEG proteins in disease control and plant tolerance. Especially, the expressions of WRKY26, MYB4, E3-KEG, PNPT1, BIP1, and BCAT1 were downregulated in $p R N A i-N t L R R-R L K$ transgenic plants at $24 \mathrm{~h}$ after inoculation with TMV. These results were helpful to demonstrate that the tolerance of Yuyan8 to TMV infection is higher than that of NC89.

\section{High methylation of the $L R R-R L K$ in NC89 is responsible for sensitivity to TMV.}

In NC89, the specific TMV-infection-responsive genes did not overlap with Yuyan8. These genes were enriched in the pyrimidine metabolism pathway, histone methylation pathway, and glycosaminoglycan degradation metabolism. Notably, most of the proteins in these metabolism pathways are related to the incidence of disease.
The expression level of most genes declined in the pyrimidine metabolism pathway. Polyribonucleotide nucleotidyltransferase 1 (PNPT1) is an RNA-binding protein that is involved in many RNA metabolic processes and catalyzes the phosphorolysis of single-stranded polyribonucleotides (Vedrenne et al. 2012; Von Ameln et al. 2012). PNPT1 was downregulated 1.83 times after TMV infection, which directly affected the expression of DdRP and then regulated the expression of H3K27 monomethyltransferase (ATXR6 and ATXR5) (Jacob et al. 2009). Histone modification is an important kind of epigenetic regulation, and abnormal histone modification might lead to many diseases (Schneider et al. 2002). In addition, thymidylate kinase (TK) is an indispensable DNA synthase for cell proliferation, and its activity level reflects the synthesis speed of DNA. TK can upregulate the expression of the branched chain amino acid transaminase (BCAT1) gene. BCATI is highly expressed in a variety of malignant tumors and is closely related to cell apoptosis, tumor cell proliferation metastasis, and invasion (Eden et al. 1996; Zhang and Han 2017). GAGs, a class of polysaccharides, are involved in the regulation of the antivirus response and cell proliferation pathways (Proudfoot et al. 2003; Raman et al. 2005; Yang and Chi 2017).

Here, it was found that the expression level of the methylated $L R R-R L K$ gene in NC89 was higher than that in Yuyan8. Therefore, it was speculated that low expression of $L R R-R L K$ in NC89 weakened signal transmission for virus invasion and recognition and, then, the intrinsic immune system was not activated in time. Subsequently, the $L R R-R L K$ gene has a large impact on histone methylation modification, the expression level of genes in pyrimidine metabolism, and the production of GAGs. Here, it had been found that silencing of $N t L R R-R L K$

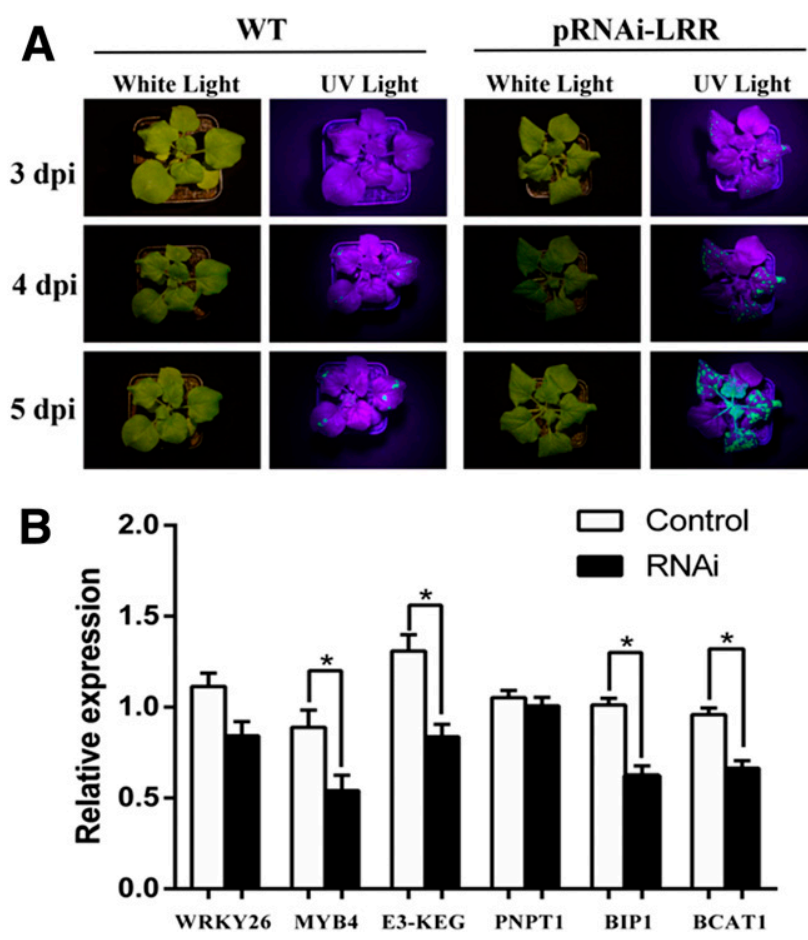

Fig. 6. Gene Nicotiana tabacum leucine-rich repeat receptor kinase (NtLRR-RLK) confers the susceptibility to tobacco mosaic virus (TMV) in Nicotiana benthamiana. A, Silencing of NtLRR-RLK in N. benthamiana significantly increased susceptibility to TMV. Green fluorescent signals representing the viral replication in $p R N A i-N t L R R-R L K$ and wild-type (WT) tobacco plants were imaged at 3, 4, and 5 days postinoculation (dpi). B, Expression levels of the critical node genes in transgenic plants and the WT. Statistical analyses were performed using Student's $t$ test (* indicates $0.01<P<0.05)$. 
significantly enhanced TMV infection. These results are helpful for understanding the susceptibility of NC89 to TMV.

Collectively, these data showed the difference in specifically expressed genes, methylation modification, and flavonoid metabolites between the two tobacco cultivars, and it is critical for us to visually demonstrate the relationship between signal transduction and biological pathways. Meanwhile, the intensive study of the nodes of proteins associated with these biological pathways also provides new ideas for protecting crops from virus infection.

\section{MATERIALS AND METHODS}

\section{Materials and TMV inoculation.}

The cultivation of tobacco and the inoculation of TMV were performed as described by Wang et al. (2016). The upper leaves adjacent to the TMV inoculation site were collected at $0 \mathrm{hpi}$, 12 hpi, 24 hpi, 6 days postinfection (dpi), and 9 dpi for RNA or DNA extraction.

\section{RNA isolation and sequencing.}

The leaves of Yuyan8 and NC89 at 0 hpi and Yd and Nd at 24 hpi were collected for RNA sequencing. Each sample was taken from a mixture of leaves from the same position on three tobacco plants. Total RNA was extracted using an Agilent plant RNA isolation kit. The quality of all samples was considered good when the optical density (OD) ratios of 260/280 ranged from 1.9 to 2.1 and the RNA integrity number was greater than 8 . The preparation and sequencing of the RNA-seq library were performed by the Shanghai Biotechnology Corporation, China. The library was sequenced on the Illumina sequencing (HiSeq 2500) platform using a paired-end $2 \times 100$ nucleotides multiplex sequencing approach.

\section{Transcript assembly and analysis.}

All processed reads from the four libraries were mixed to pool the reads and de novo assembled using CLC Genomics Workbench (version 6.0.4) (Bräutigam et al. 2011; Garg et al. 2011; Su et al. 2011). The word size was 45 and the minimum contig length was 300 . The final assembled unigene sequences were aligned by BLASTx (E-value < $1.0 \mathrm{e}-5)$ to public protein databases, including the UniProt database and SwissProt database. The transcript sequences were also aligned with the Arabidopsis thaliana, Solanum tuberosum, S. demissum, and S. lycopersicum protein databases (Phytozome portal). KEGG pathway analyses were performed using the online KEGG automatic server. The gene expression levels were calculated as fragments per kilobase of exon per million mapped fragments (Trapnell et al. 2010). A false discovery rate $<0.05$ and fold-change $\geq 2$ were used as thresholds to define the significant differences in transcript abundance.

\section{qRT-PCR validation.}

The leaves of Yuyan8 and NC89 at 0 and 24 hpi were collected for qPCR analysis. Each treatment had five biological replicates. Total RNA was reverse-transcribed using SuperScript III reverse transcription. RT-PCR was performed using the iQ5 RT-PCR detection system. The program was as follows: $95^{\circ} \mathrm{C}$ for $15 \mathrm{~s}$, followed by 40 cycles of $95^{\circ} \mathrm{C}$ for $15 \mathrm{~s}$, and then annealed at $60^{\circ} \mathrm{C}$ for $30 \mathrm{~s}$. NtActin was used as an internal control. Three replicates were performed for each example. Relative amounts of mRNA were determined with the cycle threshold $(\mathrm{Ct})$ method. The fold change was calculated using the $2^{-\Delta \Delta} \mathrm{Ct}$ method, and error bars represented the standard deviation of the mean. Statistical analysis was performed with
Student's $t$ test. A value of $P<0.05$ was considered significant. The primers for RT-PCR are listed in Supplementary Table S6.

\section{Liquid chromatography tandem mass spectrometry metabolite profiling and identification.}

The leaf metabolite extraction and liquid chromatography tandem mass spectrometry (MS/MS) protocols were the same as those previously reported (Fu et al. 2016). The leaves of Yuyan8 and NC89 at 0 and 24 hpi were collected for metabolomic analysis. The MOL files of the potential flavonoids were downloaded from the KEGG and PubChem databases, and the theoretical fragmentation patterns of these substances were then compared with practical MS/MS spectra to identify potential flavonoids. Each sample had five biological replicates for leaf metabolite analysis.

\section{MSAP analysis.}

The leaves of Yuyan8 and NC89 at 0 hpi, 12 hpi, 24 hpi, 6 dpi, and 9 dpi were collected for MSAP analysis. Each sample had three biological replicates. The MSAP, electrophoresis, and autoradiography procedures were performed according to the protocols of Xiong et al. (1999) and Peraza-Echeverria et al. (2001), with slight modifications. The PCR products were separated on $6 \%$ sequencing gels and visualized with silver staining (Chalhoub et al. 1997). The sequence information for adapters and primers of preamplification and selective amplification are provided in Supplementary Table $\mathrm{S} 4$. The protocols for cloning and characterization assays of the differentially amplified DNA fragments were the same as those reported previously (Sha et al. 2005). Briefly, the amplified fragments were excised from the gel with a razor blade. The eluted DNA was amplified with the same primers under the conditions used for selective amplification. The sequences were obtained by cloning the fragments in the T-vector and were compared with nucleotide sequences in NCBI databases using BLAST.

\section{Cloning and characterization of the differentially amplified DNA fragments bisulfite sequencing PCR (BSP).}

The genomic DNA of leaves was treated for bisulfate modification according to the combined bisulfite restriction analysis assay, as described previously (Xiong and Laird 1997). The bisulfite-modified DNA was purified using the Gene Clean kit and stored at $-80^{\circ} \mathrm{C}$. Analysis of the LRR receptor-like serine/threonine-protein kinase $\mathrm{CpG}$ loci and primer design of the amplified fragment sequence was as follows:

ACATGCCAAATGAGAGCTTAGACAAGTTGCTACA TTCGCGAGATTATTGTTTAAATATAATGCAGAGATTGAA TATCATGGTTGATGTTGCATCTGCTCTAGAATATCTCCA TCATGGTTACTCAGTACCGGTTATTCACTGTGATCTGAA GCCTAGCAATGTGTTACTTGACAATGACATGGTGGGAC ACCTAACTGACTTTGGCATTGCTAAACTTTTGACTAAGG AAGAATCTATTGCTCACACTACAACCTTTGCCACTATTGG TTACATTGCTCCAGGTGAATTCTTTATTTAACTTTGTAA CAATGTAATTGATTAACGCAACAACAACAACAACATACG CAGTGTAATCCCAAA AGTGGGGT

Sections in bold indicate designed primer position. The middle part was the sequence to be evaluated. Underlined italicized font represents $\mathrm{CpG}$ loci, and their positions in the sequence were sequentially $37,39,130,324$, and 345 .

A DNA fragment of $375 \mathrm{bp}$, including five cytosines of $\mathrm{CpG}$ loci, was amplified by PCR. The protocol for bisulfite sequencing PCR was reported previously (Ehrlich et al. 2010), with the modification of reaction conditions as described by Zhong et al. (2009). 


\section{Protein interaction relationship.}

A protein-protein interaction network was constructed and analyzed by Cytoscape 3.6.1. The network was queried by STRING database 10.5 (Szklarczyk et al. 2011) and implemented with the Centiscape plugin of Cytoscape software that determines the degree of each node (Scardoni et al. 2009). In such a network, proteins (nodes) are linked (edges) to identify the interaction between them. The size of each node indicates the amount of correlated genes. The colors of nodes represent different metabolic pathways.

\section{Generation of transgenic plants.}

$N$. benthamiana plants were transformed with $p R N A i-L R R$ using Agrobacterium-mediated leaf disc transformation (Horsch et al. 1989).Transgenic plants were selected using kana (50 mg/liter) and confirmed by PCR.

\section{TMV inoculation.}

The transgenic tobacco plants and wild-type tobacco were also cultivated in the same growing condition $\left(25^{\circ} \mathrm{C}\right.$, cycle of $16 \mathrm{~h}$ of light and $8 \mathrm{~h}$ of darkness), propagated, and transplanted at the same time and served as controls for TMV inoculation. Agrobacterium cultures at an OD at $600 \mathrm{~nm}=1.0$ containing TMV-GFP plasmids was infiltrated onto the leaf of six-leaf stage plants using a 1-ml needleless syringe. Each experiment was repeated three times with at least three independent plants per time. GFP imaging was done using UV illumination and photographs were taken using a Nikon camera.

\section{ACKNOWLEDGMENTS}

We thank Y. Liu (Tsinghua University, Beijing, China) for providing the pSPDK661 (TMV-GFP) vector.

\section{AUTHOR RECOMMENDED INTERNET RESOURCES}

China National GeneBank database: https://db.cngb.org

KEGG automatic server: https://www.genome.jp/tools/kaas

Phytozome portal: https://phytozome.jgi.doe.gov/pz/portal.html

STRING database 10.5: https://string-db.org/cgi/input.pl

\section{LITERATURE CITED}

Adachi, H., Nakano, T., Miyagawa, N., Ishihama, N., Yoshioka, M., Katou, Y., Yaeno, T., Shirasu, K., and Yoshioka, H. 2015. WRKY transcription factors phosphorylated by MAPK regulate a plant immune NADPH oxidase in Nicotiana benthamiana. Plant Cell 27:2645-2663.

Agarwal, P., Reddy, M. P., and Chikara, J. 2011. WRKY: Its structure, evolutionary relationship, DNA-binding selectivity, role in stress tolerance and development of plants. Mol. Biol. Rep. 38:3883-3896.

Akimoto, K., Katakami, H., Kim, H. J., Ogawa, E., Sano, C. M., Wada, Y., and Sano, H. 2007. Epigenetic inheritance in rice plants. Ann. Bot. 100: 205-217.

Aparicio, F., Thomas, C. L., Lederer, C., Niu, Y., Wang, D., and Maule, A. J. 2005. Virus induction of heat shock protein 70 reflects a general response to protein accumulation in the plant cytosol. Plant Physiol. 138: 529-536.

Bergman, M., Perelman, A., Dubinsky, Z., and Grossman, S. 2003. Scavenging of reactive oxygen species by a novel glucurinated flavonoid antioxidant isolated and purified from spinach. Phytochemistry 62: 753-762.

Bhattarai, K. K., Atamian, H. S., Kaloshian, I., and Eulgem, T. 2010. WRKY72-type transcription factors contribute to basal immunity in tomato and Arabidopsis as well as gene-for-gene resistance mediated by the tomato $R$ gene $M i-1$. Plant J. 63:229-240.

Boyko, A., Kathiria, P., Zemp, F. J., Yao, Y., Pogribny, I., and Kovalchuk, I. 2007. Transgenerational changes in the genome stability and methylation in pathogen-infected plants: (Virus-induced plant genome instability). Nucleic Acids Res. 35:1714-1725.

Bräutigam, A., Mullick, T., Schliesky, S., and Weber, A. P. M. 2011. Critical assessment of assembly strategies for non-model species mRNA-Seq data and application of next-generation sequencing to the comparison of $\mathrm{C}_{3}$ and $\mathrm{C}_{4}$ species. J. Exp. Bot. 62:3093-3102.

Bravo, R., Parra, V., Gatica, D., Rodriguez, A. E., Torrealba, N., Paredes, F., Wang, Z. V., Zorzano, A., Hill, J. A., Jaimovich, E., Quest, A. F., and Lavandero, S. 2013. Endoplasmic reticulum and the unfolded protein response: Dynamics and metabolic integration. Int. Rev. Cell Mol. Biol. 301:215-290.

Chalhoub, B. A., Thibault, S., Laucou, V., Rameau, C., Höfte, H., and Cousin, R. 1997. Silver staining and recovery of AFLP amplification products on large denaturing polyacrylamide gels. Biotechniques 22: 216-218, 220.

Choi, C., Hwang, S. H., Fang, I. R., Kwon, S. I., Park, S. R., Ahn, I., Kim, J. B., and Hwang, D. J. 2015. Molecular characterization of Oryza sativa WRKY6, which binds to W-box-like element 1 of the Oryza sativa pathogenesis-related (PR) 10a promoter and confers reduced susceptibility to pathogens. New Phytol. 208:846-859.

Das, P. P., Chua, G. M., Lin, Q., and Wong, S. M. 2019. iTRAQ-based analysis of leaf proteome identifies important proteins in secondary metabolite biosynthesis and defence pathways crucial to cross-protection against TMV. J. Proteomics 196:42-56.

Datta, R., Kumar, D., Sultana, A., Hazra, S., Bhattacharyya, D., and Chattopadhyay, S. 2015. Glutathione regulates 1-aminocyclopropane1-carboxylate synthase transcription via WRKY33 and 1aminocyclopropane-1-carboxylate oxidase by modulating messenger RNA stability to induce ethylene synthesis during stress. Plant Physiol. 169:2963-2981.

Dodds, P. N., and Rathjen, J. P. 2010. Plant immunity: Towards an integrated view of plant-pathogen interactions. Nat. Rev. Genet. 11:539-548.

Duplan, V., and Rivas, S. 2014. E3 ubiquitin-ligases and their target proteins during the regulation of plant innate immunity. Front. Plant Sci. 5:42.

Eden, A., Simchen, G., and Benvenisty, N. 1996. Two yeast homologs of $E C A 39$, a target for c-Myc regulation, code for cytosolic and mitochondrial branched-chain amino acid aminotransferases. J. Biol. Chem. 271: 20242-20245.

Ehrlich, S., Weiss, D., Burghardt, R., Infante-Duarte, C., Brockhaus, S., Muschler, M. A., Bleich, S., Lehmkuhl, U., and Frieling, H. 2010. Promoter specific DNA methylation and gene expression of POMC in acutely underweight and recovered patients with anorexia nervosa. J. Psychiatr. Res. 44:827-833.

Feng, L., Zhang, H. W., and Huang, R. F. 2012. Research progress on LRR receptor-like protein kinase in plant. J. Agric. Sci. Technol. 14:43-48.

Finnegan, E. J., Brettell, R. I. S., and Dennis, E. S. 1993. The role of DNA methylation in the regulation of plant gene expression. Pages 218-261 in: DNA Methylation: Molecular Biology and Biological Significance. EXS, Vol. 64. J.-P. Jost and H.-P. Saluz, eds. Birkhäuser Verlag, Basel, Switzerland.

Fu, B., Ji, X., Zhao, M., He, F., Wang, X., Wang, Y., Liu, P., and Niu, L. 2016. The influence of light quality on the accumulation of flavonoids in tobacco (Nicotiana tabacum L.) leaves. J. Photochem. Photobiol. B 162: 544-549.

Gao, L.-L., and Xue, H.-W. 2012. Global analysis of expression profiles of rice receptor-like kinase genes. Mol. Plant 5:143-153.

Garg, R., Patel, R. K., Tyagi, A. K., and Jain, M. 2011. De novo assembly of chickpea transcriptome using short reads for gene discovery and marker identification. DNA Res. 18:53-63.

Gu, Y., and Innes, R. W. 2011. The KEEP ON GOING protein of Arabidopsis recruits the ENHANCED DISEASE RESISTANCE1 protein to trans-Golgi network/early endosome vesicles. Plant Physiol. 155: 1827-1838

Han, Y., Luo, Y., Qin, S., Xi, L., Wan, B., and Du, L. 2014. Induction of systemic resistance against tobacco mosaic virus by Ningnanmycin in tobacco. Pestic. Biochem. Physiol. 111:14-18.

Horsch, R. B., Fry, J., Hoffmann, N., Neidermeyer, J., Rogers, S. G., Fraley, R. T. 1989. Leaf disc transformation. In: Plant Molecular Biology Manual. S. B. Gelvin, R. A. Schilperoort, D. P. S. Verma. eds. Springer, Dordrecht, The Netherlands.

Huang, H. T., Hao, F. S., Wei, Y. F., Qi, Z., Yang, L. J., and Liu, W. Q. 2017. Relationship between glutathione metabolism and TMV resistance in tobacco. Agric. Biotechnol. 6:1-5.

Jacob, Y., Feng, S., LeBlanc, C. A., Bernatavichute, Y. V., Stroud, H., Cokus, S., Johnson, L. M., Pellegrini, M., Jacobsen, S. E., and Michaels, S. D. 2009. ATXR5 and ATXR6 are H3K27 monomethyltransferases required for chromatin structure and gene silencing. Nat. Struct. Mol. Biol. 16:763-768.

Li, J., Wang, J., Wang, N., Guo, X., and Gao, Z. 2015. GhWRKY 44 , a WRKY transcription factor of cotton, mediates defense responses to pathogen infection in transgenic Nicotiana benthamiana. Plant Cell Tissue Organ Cult. 121:127-140. 
Ohta, M., Wakasa, Y., Takahashi, H., Hayashi, S., Kudo, K., and Takaiwa, F 2013. Analysis of rice ER-resident J-proteins reveals diversity and functional differentiation of the ER-resident Hsp70 system in plants. J. Exp. Bot. 64:5429-5441.

Padmanabhan, M., Cournoyer, P., and Dinesh-Kumar, S. P. 2009. The leucine-rich repeat domain in plant innate immunity: A wealth of possibilities. Cell. Microbiol. 11:191-198.

Padmanabhan, M. S., Ma, S., Burch-Smith, T. M., Czymmek, K., Huijser, P., and Dinesh-Kumar, S. P. 2013. Novel positive regulatory role for the SPL6 transcription factor in the N TIR-NB-LRR receptor-mediated plant innate immunity. PLoS Pathog. 9:e1003235.

Peraza-Echeverria, S., Herrera-Valencia, V. A., and Kay, A. J. 2001 Detection of DNA methylation changes in micropropagated banana plants using methylation-sensitive amplification polymorphism (MSAP). Plant Sci. 161:359-367.

Proudfoot, A. E., Handel, T. M., Johnson, Z., Lau, E. K., LiWang, P., ClarkLewis, I., Borlat, F., Wells, T. N. C., and Kosco-Vilbois, M. H. 2003. Glycosaminoglycan binding and oligomerization are essential for the in vivo activity of certain chemokines. Proc. Natl. Acad. Sci. U.S.A. 100: 1885-1890.

Raman, R., Sasisekharan, V., and Sasisekharan, R. 2005. Structural insights into biological roles of protein-glycosaminoglycan interactions. Chem. Biol. 12:267-277.

Ren, X.-J., Huang, W.-D., Li, W.-Z., and Yu, D.-Q. 2010. Tobacco transcription factor WRKY4 is a modulator of leaf development and disease resistance. Biol. Plant. 54:684-690.

Robatzek, S., Chinchilla, D., and Boller, T. 2006. Ligand-induced endocytosis of the pattern recognition receptor FLS2 in Arabidopsis. Genes Dev. 20:537-542.

Scardoni, G., Petterlini, M., and Laudanna, C. 2009. Analyzing biological network parameters with CentiScaPe. Bioinformatics 25:2857-2859.

Schneider, R., Bannister, A. J., and Kouzarides, T. 2002. Unsafe SETs: Histone lysine methyltransferases and cancer. Trends Biochem. Sci. 27:396-402.

Sello, S., Perotto, J., Carraretto, L., Szabò, I., Vothknecht, U. C., and Navazio, L. 2016. Dissecting stimulus-specific $\mathrm{Ca}^{2+}$ signals in amyloplasts and chloroplasts of Arabidopsis thaliana cell suspension cultures. J. Exp. Bot. 67:3965-3974.

Serrano, M., Kanehara, K., Torres, M., Yamada, K., Tintor, N., Kombrink, E., Schulze-Lefert, P., and Saijo, Y. 2012. Repression of sucrose/ultraviolet B light-induced flavonoid accumulation in microbeassociated molecular pattern-triggered immunity in Arabidopsis. Plant Physiol. 158:408-422.

Sha, A. H., Lin, X. H., Huang, J. B., and Zhang, D. P. 2005. Analysis of DNA methylation related to rice adult plant resistance to bacterial blight based on methylation-sensitive AFLP (MSAP) analysis. Mol. Genet. Genomics 273:484-490.

Singh, A., Gupta, R., and Pandey, R. 2017. Exogenous application of rutin and gallic acid regulate antioxidants and alleviate reactive oxygen generation in Oryza sativa L. Physiol. Mol. Biol. Plants 23:301-309.

Su, C.-L., Chao, Y.-T., Alex Chang, Y.-C., Chen, W.-C., Chen, C.-Y., Lee, A.-Y., Hwa, K. T., and Shih, M.-C. 2011. De novo assembly of expressed transcripts and global analysis of the Phalaenopsis aphrodite transcriptome. Plant Cell Physiol. 52:1501-1514.

Szklarczyk, D., Franceschini, A., Kuhn, M., Simonovic, M., Roth, A., Minguez, P., Doerks, T., Stark, M., Muller, J., Bork, P., Jensen, L. J., and von Mering, C. 2011. The STRING database in 2011: Functional interaction networks of proteins, globally integrated and scored. Nucleic Acids Res. 39:D561-D568.

Takken, F. L., Albrecht, M., and Tameling, W. I. 2006. Resistance proteins: Molecular switches of plant defence. Curr. Opin. Plant Biol. 9:383-390.

Tanaka, K., Masuda, R., Sugimoto, T., Kawamura, Y., and Kuboi, T. 2014. An $\mathrm{H}_{2} \mathrm{O}_{2}$-decomposing system in cultured tobacco cells. J. Agric. Chem. Soc. Jpn. 54:2003-2008.

Tang, D., Wang, G., and Zhou, J. M. 2017. Receptor kinases in plantpathogen interactions: More than pattern recognition. Plant Cell 29: 618-637.

Torii, K. U. 2004. Leucine-rich repeat receptor kinases in plants: Structure, function, and signal transduction pathways. Int. Rev. Cytol. 234:1-46.
Trapnell, C., Williams, B. A., Pertea, G., Mortazavi, A., Kwan, G., van Baren, M. J., Salzberg, S. L., Wold, B. J., and Pachter, L. 2010. Transcript assembly and quantification by RNA-Seq reveals unannotated transcripts and isoform switching during cell differentiation. Nat. Biotechnol. 28:511-515.

van Verk, M. C., Neeleman, L., Bol, J. F., and Linthorst, H. J. M. 2011. Tobacco transcription factor NtWRKY12 interacts with TGA2.2 in vitro and in vivo. Front. Plant Sci. 2:32.

van Verk, M. C., Pappaioannou, D., Neeleman, L., Bol, J. F., and Linthorst, H. J. M. 2008. A novel WRKY transcription factor is required for induction of $P R-1 a$ gene expression by salicylic acid and bacterial elicitors. Plant Physiol. 146:1983-1995.

Vedrenne, V., Gowher, A., De Lonlay, P., Nitschke, P., Serre, V., Boddaert, N., Altuzarra, C., Mager-Heckel, A. M., Chretien, F., Entelis, N., Munnich, A., Tarassov, I., and Rötig, A. 2012. Mutation in PNPT1, which encodes a polyribonucleotide nucleotidyltransferase, impairs RNA import into mitochondria and causes respiratory-chain deficiency. Am. J. Hum. Genet. 91:912-918.

Verchot, J. 2014. The ER quality control and ER associated degradation machineries are vital for viral pathogenesis. Front. Plant Sci. 5:66.

Verchot, J. 2016. How does the stressed out ER find relief during virus infection? Curr. Opin. Virol. 17:74-79.

von Ameln, S., Wang, G., Boulouiz, R., Rutherford, M. A., Smith, G. M., Li, Y., Pogoda, H. M., Nürnberg, G., Stiller, B., Volk, A. E., Borck, G., Hong, J. S., Goodyear, R. J., Abidi, O., Nürnberg, P., Hofmann, K., Richardson, G. P., Hammerschmidt, M., Moser, T., Wollnik, B., Koehler, C. M., Teitell, M. A., Barakat, A., and Kubisch, C. 2012. A mutation in PNPT1, encoding mitochondrial-RNA-import protein PNPase, causes hereditary hearing loss. Am. J. Hum. Genet. 91:919-927.

Wada, Y., Miyamoto, K., Kusano, T., and Sano, H. 2004. Association between up-regulation of stress-responsive genes and hypomethylation of genomic DNA in tobacco plants. Mol. Genet. Genomics 271:658-666.

Wang, A. 2015. Dissecting the molecular network of virus-plant interactions: The complex roles of host factors. Annu. Rev. Phytopathol. 53:45-66.

Wang, A., and Krishnaswamy, S. 2012. Eukaryotic translation initiation factor 4E-mediated recessive resistance to plant viruses and its utility in crop improvement. Mol. Plant Pathol. 13:795-803

Wang, J., Wang, H. Y., Xia, X. M., Li, P. P., and Wang, K. Y. 2013. Inhibitory effect of esterified lactoferin and lactoferrin against tobacco mosaic virus (TMV) in tobacco seedlings. Pestic. Biochem. Physiol. 105:62-68.

Wang, J., Wang, X. R., Zhou, Q., Yang, J. M., Guo, H. X., Yang, L. J., and Liu, W. Q. 2016. iTRAQ protein profile analysis provides integrated insight into mechanisms of tolerance to TMV in tobacco (Nicotiana tabacum). J. Proteomics 132:21-30.

Williams, B., Verchot, J., and Dickman, M. B. 2014. When supply does not meet demand-ER stress and plant programmed cell death. Front. Plant Sci. 5:211.

Xiong, L. Z., Xu, C. G., Saghai Maroof, M. A., and Zhang, Q. 1999. Patterns of cytosine methylation in an elite rice hybrid and its parental lines, detected by a methylation-sensitive amplification polymorphism technique. Mol. Gen. Genet. 261:439-446.

Xiong, Z., and Laird, P. W. 1997. COBRA: A sensitive and quantitative DNA methylation assay. Nucleic Acids Res. 25:2532-2534.

Yang, J., and Chi, L. 2017. Characterization of structural motifs for interactions between glycosaminoglycans and proteins. Carbohydr. Res. 452:54-63.

Ye, C., Dickman, M. B., Whitham, S. A., Payton, M., and Verchot, J. 2011. The unfolded protein response is triggered by a plant viral movement protein. Plant Physiol. 156:741-755.

Zhang, L., and Han, J. 2017. Branched-chain amino acid transaminase 1 (BCAT1) promotes the growth of breast cancer cells through improving mTOR-mediated mitochondrial biogenesis and function. Biochem. Biophys. Res. Commun. 486:224-231.

Zhong, L., Xu, Y.-H., and Wang, J.-B. 2009. DNA-methylation changes induced by salt stress in wheat. Afr. J. Biotechnol. 8:6201-6207.

Zipfel, C. 2014. Plant pattern-recognition receptors. Trends Immunol. 35: 345-351. 